\title{
Analysis of Transients in a 4-Level Flying Capacitor Converter: Time Domain Approach. Part 2: Small Normalised Voltage Command
}

\author{
Boris Reznikov ${ }^{1, *}$, Alex Ruderman², Valentina Galanina ${ }^{3}$ \\ 1 GS Group, St. Petersburg, Russia \\ ${ }^{2}$ Department of Electrical and Computer Engineering, Nazarbayev University, Nur-Sultan, Kazakhstan, Israel \\ ${ }^{3}$ Department of Applied Mathematics, State University of Aerospace Instrumentation, St. Petersburg, Russia
}

Received: October 03, 2019; Accepted: January 05, 2020

\begin{abstract}
A 4-level flying capacitor converter (FCC) operation is considered on a base of discrete state-space model. A transition matrix is obtained for a pulse width modulation (PWM) period for small normalised voltage command values $[0,1 / 3]$. The transition matrix elements are expanded into power series by small parameters. The matrix eigenvalues are presented in the form of power series as well. Six separate transients are constructed for six possible initial FCC states on a PWM period. Inductor current and capacitors' voltage transients are found for the voltage source power-up as the arithmetic average of the six separate transients. Finally, the discrete solutions are replaced by equivalent continuous ones. Simple and accurate formulas for inductor current and capacitors' voltage transients demonstrate good agreement with simulation results.
\end{abstract}

Keywords: 4-level DC-DC converter • flying capacitor • natural balancing • transients

\section{Introduction}

The first part of analytical study of transients in a 4-level DC-DC flying capacitor converter (FCC) for large reference voltages $D$ is presented in Reznikov et al. (2019 submitted to). In that study, the approach described in details is used for transient matrix calculation with elements as series by powers of small parameter, calculation of eigenvalues and eigenvectors of this matrix and, finally, calculation of transients of the converter. As a result, simple and accurate formulas were obtained for $1 / 3 \leq D \leq 1$. In this paper, the analytical study is continued for a range of small $D(0 \leq D \leq 1 / 3)$, thus completing the analytical description of 4-level DC-DC FCC. As in Reznikov et al. (2019 submitted to), the time-domain approach will be taken as a basis of analytical research. The theoretical essence of this approach was described in detail in Reznikov et al. (2019 submitted to). Therefore, here some explanations will be omitted with references to the previous papers provided instead. The goal of this paper as in Reznikov et al. (2019 submitted to) is to obtain simple and accurate formulas for average natural balancing transients in case of small $D$ values as functions of FCC circuit and load parameters and also pulse width modulation (PWM) period.

\section{Discrete Model Construction}

Figure 1a presents a 4-level DC-DC FCC circuit. All the following analysis is based on the same assumptions accepted for a 4-level FCC in case of large $D$ (Reznikov et al., 2019 submitted to), namely, the load is considered 
as a series connection of inductor and resistor, the bi-directional switches have zero resistance in the conductive state (ON) and infinite in the open-circuit one (OFF), and switching rise and fall times equal zero.

Just as in the case of large $D$ (Reznikov et al., 2019 submitted to), the FCC operation will be analysed only for positive $D$ values due to the symmetry considerations. 4-level FCC balancing dynamics depend on modulation strategy. The classic realisation of carrier-based PWM is described in detail in Reznikov and Ruderman (2009).

The modulation strategy is determined by the switching algorithm of the complementary switch pairs $\mathrm{S} 1-S 1, \mathrm{~S} 2-S 2$ and $\mathrm{S} 3-S 3$. This algorithm is the same for both ranges of large and small $D$. A PWM period $T_{P W M}$ is divided into six successive time intervals $\Delta t_{1}, \Delta t_{2}, \Delta t_{3}, \Delta t_{4}, \Delta t_{5}, \Delta t_{6}$. The intervals with even (odd) indices have the same duration, that is $\Delta t_{2}=\Delta t_{4}=\Delta t_{6}\left(\Delta t_{1}=\Delta t_{3}=\Delta t_{5}\right)$. Each interval index corresponds to its own equivalent circuit (topology) with the number equal to the interval index. The topologies with odd numbers are presented in Figure $1 \mathrm{~b}$ and those with even ones in Figure 1c. The difference from the case of large $D$ is in the fact that, instead of one topology for all even indices, three different topologies participate in FCC operation in the case of small $D$.

The topologies are defined by the following switch states. In Topology 1, switches S2 and S3 are ON, and switch S1 is OFF. In Topology 3, switches S1 and S3 are ON, and switch S2 is OFF. In Topology 5, switches S1 and S2 are ON, and switch S3 is OFF. In Topology 2, switch S3 is ON, and switches S1 and S2 are OFF. In Topology 4, switch S1 is ON, and switches S2 and S3 are OFF. In Topology 6, switch S2 is ON, and switches S1 and S3 are OFF.

Similar to the large $D$ case, space vector $X(t)=\left(i_{L}(t) v_{C_{1}}(t) v_{C_{2}}(t)\right)^{T}$ is introduced and the matrix relation for arbitrary time interval $\Delta t_{k}$ is written as

$$
X\left(t+\Delta t_{k}\right)=A_{k}\left(\Delta t_{k}\right) X(t)+B_{k}\left(\Delta t_{k}\right) V / 2
$$

where matrices $A_{k}$ and vectors $B_{k}$ relate to their corresponding topologies and time interval durations.

Associating subsequently equation (1) with each of six PWM period $T_{P W M}$ intervals and using the continuation of vector $X$ coordinates at the switching moments lead to

$$
X^{(1)}\left(t+T_{P W M}\right)=A^{(1)} X^{(1)}(t)+B^{(1)} V / 2
$$

(a)

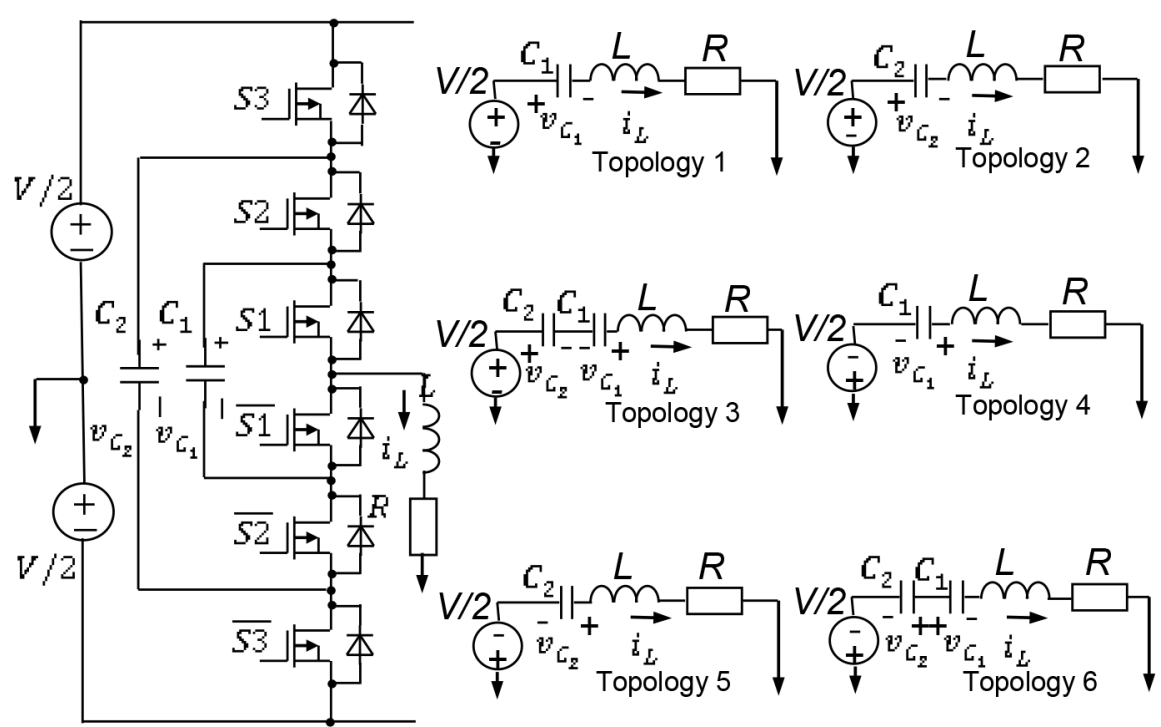

Fig. 1. 4-level FCC circuit (a) and its switched topologies for the small $D$ case (b and c). 
where transient matrix $A^{(1)}$ and vector $B^{(1)}$ are defined as follows:

$$
\begin{aligned}
& A^{(1)}=A_{6} A_{5} A_{4} A_{3} A_{2} A_{1} \\
& B^{(1)}=A_{6}\left(A_{5}\left(A_{4}\left(A_{3}\left(A_{2} B_{1}+B_{2}\right)+B_{3}\right)+B_{4}\right)+B_{5}\right)+B_{6}
\end{aligned}
$$

Equations (3) and (4) are written down assuming that the PWM period starts with the time interval 1 as pointed by the superscript in parentheses.

Similar to the large $D$ range, the proposed analysis ignores the FCC oscillating behaviour inside the PWM period and is aimed at describing the average behaviour in "long" discrete time $t_{k}=k T_{P W M}$. In this way, equation (2) may be rewritten as

$$
X^{(1)}\left(t_{k+1}\right)=A^{(1)} X^{(1)}\left(t_{k}\right)+B^{(1)} V / 2, t_{k+1}=t_{k}+T_{P W M}
$$

As shown in Reznikov and Ruderman (2009), the time interval durations for small $D$ become

$$
\begin{aligned}
& \Delta t_{1}=\Delta t_{3}=\Delta t_{5}=\frac{1 / 3+|D|}{2} T_{\mathrm{PWM}} \\
& \Delta t_{2}=\Delta t_{4}=\Delta t_{6}=\frac{1 / 3-|D|}{2} T_{\mathrm{PWM}}
\end{aligned}
$$

Let $\omega_{1}=\sqrt{\left(1 / L C_{1}\right)-\alpha^{2}}, \omega_{2}=\sqrt{\left(1 / L C_{2}\right)-\alpha^{2}}$ and $\omega_{3}=\sqrt{\left(\left(C_{2}+C_{1}\right) / L C_{2} C_{1}\right)-\alpha^{2}}$ with $\alpha=R /(2 L)$ be the oscillation frequencies for topologies 1 and 4,5 and 2 and 3 and 6 , correspondingly. Then, using the reasoning similar to that for large $D$ (Reznikov et al., 2019 submitted to), in order to simplify the calculations, let us introduce the small parameter $\beta=\left(\omega_{1}(1 / 3+|D|) / 2\right) T_{\mathrm{PWM}}$. As, due to the symmetry considerations, only positive $D$ is considered, $D$ is used instead of its absolute value in the following. Just as in the case of large $D$, let us use the following designations: $s_{-}(\omega)=\cos \omega \tau-(\alpha / \omega) \sin \omega \tau, s_{+}(\omega)=\cos \omega \tau+(\alpha / \omega) \sin \omega \tau, r_{c}=C_{1} / C_{2}$. Simple transformations lead to $\omega_{2}=k_{1} \omega_{1}$ and $\omega_{3}=k_{2} \omega_{1}$, where $k_{1}=\sqrt{r_{c}\left(1+r^{2}\right)-r^{2}}$ and $k_{2}=\sqrt{r_{c}\left(1+r^{2}\right)+1}$.

Next, denote $w_{-}(k, \tau)=\cos k \tau \beta-(r / k) \sin k \tau \beta$ and $w_{+}(k, \tau)=\cos k \tau \beta+(r / k) \sin k \tau \beta$. Then, the above designations allow to compactly represent matrices $A_{k}$ and vectors $A_{k}$ as functions of $\beta$ :

$$
\begin{aligned}
& A_{1}=e^{-r \beta}\left(\begin{array}{ccc}
w_{-}(1,1) & -\frac{\sin \beta}{\omega_{1} L} & 0 \\
\omega_{1} L\left(1+r^{2}\right) \sin \beta & w_{+}(1,1) & 0 \\
0 & 0 & e^{r \beta}
\end{array}\right) \\
& A_{3}=e^{-r \beta}\left(\begin{array}{ccc}
w_{-}\left(k_{2}, 1\right) & \frac{\sin k_{2} \beta}{k_{1} \omega_{1} L} & -\frac{\sin k_{2} \beta}{k_{1} \omega_{1} L} \\
-\frac{\omega_{1}}{k_{1}} L\left(1+r^{2}\right) \sin k_{2} \beta & \frac{w_{+}\left(k_{2}, 1\right)+r_{c} e^{r \beta}}{1+r_{c}} & \frac{-w_{+}\left(k_{2}, 1\right)+e^{r \beta}}{1+r_{c}} \\
\frac{\omega_{1} r_{c}}{k_{1}} L\left(1+r^{2}\right) \sin k_{2} \beta & r_{c} \frac{-w_{+}\left(k_{2}, 1\right)+e^{r \beta}}{1+r_{c}} & \frac{r_{c} w_{+}\left(k_{2}, 1\right)+e^{r \beta}}{1+r_{c}}
\end{array}\right)
\end{aligned}
$$


$A_{5}=e^{-r \beta}\left(\begin{array}{ccc}w_{-}\left(k_{1}, 1\right) & 0 & \frac{\sin k_{1} \beta}{k_{1} \omega_{1} L} \\ 0 & e^{r \beta} & 0 \\ \frac{-\omega_{1} r_{c}}{k_{1}} L\left(1+r^{2}\right) \sin k_{1} \beta & 0 & w_{+}\left(k_{1}, 1\right)\end{array}\right)$

$A_{2}=e^{-r \beta k_{t}}\left(\begin{array}{ccc}w_{-}\left(k_{1}, k_{t}\right) & 0 & -\frac{\sin k_{1} k_{t} \beta}{\omega_{1} L} \\ 0 & \exp \left(r \beta k_{t}\right) & 0 \\ \frac{\omega_{1} L r_{c}\left(1+r^{2}\right) \sin k_{1} k_{t} \beta}{k_{1}} & 0 & w_{+}\left(k_{1}, k_{t}\right)\end{array}\right)$

$A_{4}=e^{-r \beta k_{t}}\left(\begin{array}{ccc}w_{-}\left(1, k_{t}\right) & 0 & -\frac{\sin k_{t} \beta}{\omega_{1} L} \\ \omega_{1} L\left(1+r^{2}\right) \sin k_{t} \beta & w_{+}\left(1, k_{t}\right) & 0 \\ 0 & 0 & e^{r \beta k_{t}}\end{array}\right)$

$A_{6}=e^{-r \beta}\left(\begin{array}{ccc}w_{-}\left(k_{2}, k_{t}\right) & -\frac{\sin k_{2} k_{t} \beta}{k_{2} \omega_{1} L} & -\frac{\sin k_{2} k_{t} \beta}{k_{2} \omega_{1} L} \\ \frac{\omega_{1}}{k_{2}} L\left(1+r^{2}\right) \sin k_{2} k_{t} \beta & \frac{w_{+}\left(k_{2}, k_{t}\right)+r_{c} e^{r \beta}}{1+r_{c}} & \frac{-w_{+}\left(k_{2}, k_{t}\right)+e^{r \beta}}{1+r_{c}} \\ \frac{-\omega_{1} r_{c}}{k_{2}} L\left(1+r^{2}\right) \sin k_{2} k_{t} \beta & r_{c} \frac{-w_{+}\left(k_{2}, k_{t}\right)+e^{r \beta}}{1+r_{c}} & \frac{r_{c} w_{+}\left(k_{2}, k_{t}\right)+e^{r \beta}}{1+r_{c}}\end{array}\right)$

$B_{1}=\left(\begin{array}{c}\frac{\exp (-r \beta) \sin \beta}{\omega L} \\ 1-\exp (-r \beta) w_{+}(1,1) \\ 0\end{array}\right)$

$B_{3}=\left(\begin{array}{c}\frac{\exp (-r \beta) \sin \left(\beta k_{2}\right)}{\omega L k_{2}} \\ \frac{\exp (-r \beta) w_{+}\left(k_{2}, 1\right)-1}{1+r_{c}} \\ \frac{1-\exp (-r \beta) w_{+}\left(k_{2}, 1\right)}{1+r_{c}} r_{c}\end{array}\right)$

$B_{5}=\left(\begin{array}{c}-\frac{\exp (-r \beta) \sin \left(\beta k_{1}\right)}{\omega L k_{1}} \\ 0 \\ 1-\exp (-r \beta) w_{+}\left(k_{1}, 1\right)\end{array}\right)$ 


$$
\begin{aligned}
& B_{2}=\left(\begin{array}{c}
\frac{\exp \left(-r \beta k_{t}\right) \sin \left(\beta k_{1} k_{t}\right)}{\omega L k_{1}} \\
0 \\
1-\exp (-r \beta) w_{+}\left(k_{1}, k_{t}\right)
\end{array}\right) \\
& B_{4}=\left(\begin{array}{c}
-\frac{\exp \left(-r \beta k_{t}\right) \sin \left(\beta k_{t}\right)}{\omega L} \\
1-\exp \left(-r \beta k_{t}\right) w_{+}\left(1, k_{t}\right) \\
0
\end{array}\right) \\
& B_{6}=\left(\begin{array}{c}
-\frac{\exp \left(-r \beta k_{t}\right) \sin \left(\beta k_{2} k_{t}\right)}{\omega L k_{2}} \\
\frac{\exp \left(-r \beta k_{t}\right) w_{+}\left(k_{2}, k_{t}\right)-1}{1+r_{c}} \\
\frac{1-\exp \left(-r \beta k_{t}\right) w_{+}\left(k_{2}, k_{t}\right)}{1+r_{c}} r_{c}
\end{array}\right)
\end{aligned}
$$

\section{Eigenvalues}

Expansion of the matrix $A_{i}$ elements into a series of $\beta$ followed by their multiplication according to equation (3) and expansion of elements of the matrix product (3) in the form of series of $\beta$ yield

$$
\begin{aligned}
& A^{(1)}=\left\{a_{i j}^{(1)}\right\}_{\substack{i=1 \\
j=1}}^{3} \\
& a_{11}^{(1)}=1-\frac{12 r \beta}{1+3 D}+\frac{72 r^{2} \beta^{2}}{(1+3 D)^{2}}+\frac{4 r\left(\left(1+r^{2}\right)\left(27 D^{2}-2 r_{c}(9 D-7)\right)-211 r^{2}+5\right) \beta^{3}}{3(1+3 D)^{3}}+\ldots \\
& a_{12}^{(1)}=\frac{24 r D \beta^{2}}{\omega L(1+3 D)^{2}}-\frac{3\left(r_{c}\left(1+r^{2}\right)(D-1)\left(3 D^{2}-1\right)-8 r^{2}\left(3 D^{2}-6 D-1\right)\right) \beta^{3}}{\omega L(1+3 D)^{3}}+\ldots \\
& a_{13}^{(1)}=\frac{12 r(1-D) \beta^{2}}{\omega L(1+3 D)^{2}}-\frac{6\left(r^{2}\left(3 D^{3}+6 D^{2}-17 D+10\right)+D\left(3 D^{2}-1\right)\right) \beta^{3}}{\omega L(1+3 D)^{3}}+\ldots \\
& a_{21}^{(1)}=\frac{24 \omega L r\left(1+r^{2}\right) \beta^{2}}{(1+3 D)^{2}}+\frac{3 \omega L\left(1+r^{2}\right)\left(r_{c}\left(1+r^{2}\right)\left(3 D^{2}-1\right)(D-1)-8 r^{2}\left(3 D^{2}+6 D-1\right)\right) \beta^{3}}{(1+3 D)^{3}}+\ldots \\
& a_{22}^{(1)}=1-\frac{4 r\left(1+r^{2}\right)\left(27 D^{2}+5\right) \beta^{3}}{3(1+3 D)^{3}}+\ldots
\end{aligned}
$$




$$
\begin{aligned}
& a_{23}^{(1)}=-\frac{3\left(1+r^{2}\right)\left(3 D^{2}-1\right) \beta^{2}}{(1+3 D)^{2}}+\frac{2 r\left(1+r^{2}\right)\left(27 D^{2}-36 D+5\right) \beta^{3}}{3(1+3 D)^{3}}+\ldots \\
& a_{31}^{(1)}=-\frac{12 \omega L r_{c} r\left(1+r^{2}\right)(D-1) \beta^{2}}{(1+3 D)^{2}}+\frac{6 \omega L r_{c}\left(1+r^{2}\right)\left(r^{2}\left(3 D^{3}+6 D^{2}+7 D-14\right)+3 D\left(D^{2}-1\right)\right) \beta^{3}}{(1+3 D)^{3}}+\ldots \\
& a_{32}^{(1)}=\frac{3 r_{c}\left(1+r^{2}\right)\left(3 D^{2}-1\right) \beta^{2}}{(1+3 D)^{2}}+\frac{2 r\left(1+r^{2}\right) r_{c}\left(27 D^{2}-36 D+5\right) \beta^{3}}{3(1+3 D)^{3}}+\ldots \\
& a_{33}^{(1)}=1+\frac{8 r\left(1+r^{2}\right) r_{c}(9 D-7) \beta^{3}}{3(1+3 D)^{3}}+\ldots
\end{aligned}
$$

Matrix $A^{(1)}$ characteristic polynomial is found as $p(\lambda)=\operatorname{det}\left(A^{(1)}-\lambda E\right)$, where $E$ is a $3 \times 3$ unity matrix. The polynomial roots are matrix $A^{(1)}$ eigenvalues. As noted in Reznikov et al. (2019 submitted to), all other matrices $A^{(i)}$, $i=1,2,3,4,5$, have the same eigenvalues because they are obtained by a cyclic permutation of $A^{(1)}$ factor matrices. The characteristic polynomial becomes

$$
p(\lambda)=\lambda^{3}+g_{1} \lambda^{2}+g_{2} \lambda+g_{3}
$$

where the coefficient series expansions in $\beta$ amount to

$$
\begin{aligned}
& g_{1}=-3+\frac{12 r \beta}{1+3 D}-\frac{72 r^{2} \beta^{2}}{(1+3 D)^{2}}+\frac{288 r^{3} \beta^{3}}{3(1+3 D)^{3}}+\frac{\left(F_{1} r^{4}+F_{2} r^{2}+9 r_{c}\left(3 D^{2}-1\right)^{2}\right) \beta^{4}}{(1+3 D)^{4}}+\ldots \\
& g_{2}=3-\frac{24 r \beta}{1+3 D}+\frac{144 r^{2} \beta^{2}}{(1+3 D)^{2}}-\frac{576 r^{3} \beta^{3}}{(1+3 D)^{3}}-\frac{\left(\left(F_{1}+864\right) r^{4}+F_{2} r^{2}+9 r_{c}\left(3 D^{2}-1\right)^{2}\right) \beta^{4}}{(1+3 D)^{4}}+\ldots \\
& g_{3}=-1+\frac{12 r \beta}{1+3 D}-\frac{72 r^{2} \beta^{2}}{(1+3 D)^{2}}+\frac{288 r^{3} \beta^{3}}{(1+3 D)^{3}}-\frac{864 r^{4} \beta^{4}}{(1+3 D)^{4}}+\ldots
\end{aligned}
$$

with

$$
\begin{aligned}
& F_{1}=81 r_{c} D^{4}-198 r_{c} D^{2}-144 D^{2}+89 r_{c}-784 \\
& F_{2}=162 r_{c} D^{4}-252 r_{c} D^{2}-144 D^{2}+98 r_{c}+80
\end{aligned}
$$

Following the method described in details in Reznikov et al. (2019 submitted to), we present the polynomial roots as series expansions in $\beta$ :

$$
\lambda_{i}=u_{0}^{(i)}+u_{1}^{(i)} \beta+u_{2}^{(i)} \beta^{2}+u_{3}^{(i)} \beta^{3}+\ldots
$$

with the superscript $i$ equal to the root number. The convergence of the series (22) is justified by the same reasons as in the large $D$ case (Reznikov et al., 2019 submitted to). 
Now, substitute equation (22) into equation (18) and equate the series to zero:

$$
p\left(\lambda_{i}\right)=c_{0}^{(i)}+c_{1}^{(i)} \beta+c_{2}^{(i)} \beta^{2}+c_{3}^{(i)} \beta^{3}+c_{4}^{(i)} \beta^{4}+\ldots=0
$$

By carrying out the same operations as for large $D$ (Reznikov et al., 2019 submitted to) that is successively equating the coefficients $c_{k}^{(i)}(k=0,1,2, \ldots)$ to zero, one can obtain the values of $u_{j}^{(i)}(j=0,1,2, \ldots)$ for each of the three roots of characteristic polynomial (18). This calculation yields the following representations of the roots (or matrix $A^{(1)}$ eigenvalues):

$$
\begin{aligned}
& \lambda_{1}=1-\frac{12 r \beta}{1+3 D}+\frac{72 r^{2} \beta^{2}}{(1+3 D)^{2}}+\frac{4 r\left(\left(1+r^{2}\right)\left(9 D^{2}-5\right)\left(1+r_{c}\right)+216 r^{2}\right) \beta^{3}}{3(1+3 D)^{3}}+O\left(\beta^{4}\right) \\
& \lambda_{2,3}=1 \mp j \frac{3\left(1+r^{2}\right)\left(1-3 D^{2}\right) r_{c} \beta^{2}}{(1+3 D)^{2}}+\frac{2 r\left(1+r^{2}\right)\left(9 D^{2}-5\right)\left(1+r_{c}\right) \beta^{3}}{3(1+3 D)^{3}}+O\left(\beta^{4}\right)
\end{aligned}
$$

The complex conjugate roots $\lambda_{2}$ and $\lambda_{3}$ can be presented in the exponential form:

$$
\lambda_{2}=M \exp (j \varphi), \lambda_{3}=M \exp (-j \varphi)
$$

where the module $M$ and argument $\varphi$ series expansions are

$$
\begin{aligned}
& M=\sqrt{\operatorname{Re}^{2}\left(\lambda_{2}\right)+\operatorname{Im}^{2}\left(\lambda_{2}\right)}=1-\frac{2 r\left(1+r^{2}\right)\left(1+r_{c}\right)\left(5-9 D^{2}\right)}{3(1+3 D)^{3}} \beta^{3}+O\left(\beta^{4}\right) \\
& \varphi=\left|\operatorname{arctg}\left(\frac{\operatorname{Im}\left(\lambda_{2}\right)}{\operatorname{Re}\left(\lambda_{2}\right)}\right)\right|=\frac{3\left(1+r^{2}\right) \sqrt{r_{c}}\left(1-3 D^{2}\right)}{(1+3 D)^{2}} \beta^{2}+O\left(\beta^{4}\right)
\end{aligned}
$$

As follows from (24) and (27), the modules of all three roots are less than unity that means that the system is stable and its transients converge.

\section{Partial Transients of Natural Balancing Dynamics}

The three different eigenvalues $\lambda_{1}, \lambda_{2}$ and $\lambda_{3}$ define three eigenfunctions $\lambda_{1}^{k}, \lambda_{2}^{k}$ and $\lambda_{3}^{k}$ of the difference

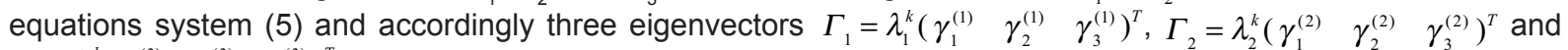
$\Gamma_{3}=\lambda_{3}^{k}\left(\gamma_{1}^{(3)} \gamma_{2}^{(3)} \gamma_{3}^{(3)}\right)^{T}$. As the eigenvectors are defined up to a constant multiplier, one of their components can be chosen arbitrarily, for example, equal to 1 . Select $\gamma_{1}^{(1)}=1, \gamma_{1}^{(2)}=1$ and $\gamma_{1}^{(3)}=1$. Then, the second and third eigenvector components are found from the system of linear equations in the matrix form:

$$
\left(\begin{array}{ccc}
a_{11}^{(1)}-\lambda_{i} & a_{12}^{(1)} & a_{13}^{(1)} \\
a_{21}^{(1)} & a_{22}^{(1)}-\lambda_{i} & a_{23}^{(1)} \\
a_{31}^{(1)} & a_{32}^{(1)} & a_{33}^{(1)}-\lambda_{i}
\end{array}\right)\left(\begin{array}{c}
1 \\
\gamma_{2}^{(i)} \\
\gamma_{3}^{(i)}
\end{array}\right)=0
$$

As the system (29) is linear dependent, any two equations can be used to find $\gamma_{2}^{(i)}$ and $\gamma_{3}^{(i)}$. Substituting $\lambda_{1}, \lambda_{2}$ and $\lambda_{3}$ in (29) and expanding the result into a series yields

$$
\gamma_{2}^{(1)}=-\frac{2 \omega L D}{1+3 D}\left(1+r^{2}\right) \beta+\frac{2 r\left(1+r^{2}\right)\left(3 D^{2}-1\right) \omega L}{(1+3 D)^{2}} \beta^{2}+\ldots
$$


Small normalised voltage command

$$
\begin{aligned}
& \gamma_{3}^{(1)}=\frac{r_{c}\left(1+r^{2}\right) \omega L(D-1)}{1+3 D} \beta-\frac{r_{c} r\left(1+r^{2}\right)(D-1) \omega L}{1+3 D} \beta^{2}+\ldots \\
& \gamma_{2}^{(2)}=\frac{\omega L(1+3 D)\left(2 D-j \sqrt{r_{c}}(D-1)\right)}{r_{c}(1-D)^{2}+4 D^{2}} \beta^{-1}+\frac{r \omega L\left(F 1 r+j \sqrt{r_{c}} F 1 i\right)}{9\left(3 D^{2}-1\right)\left(r_{c}(1-D)^{2}+4 D^{2}\right)^{2}}+\ldots \\
& \gamma_{3}^{(2)}=\frac{-\omega L(1+3 D)\left(r_{c}(D-1)+2 D j \sqrt{r_{c}}\right)}{r_{c}(1-D)^{2}+4 D^{2}} \beta^{-1}+\frac{r \omega L\left(F 2 r+2 j \sqrt{r_{c}} F 2 i\right)^{2}}{9\left(3 D^{2}-1\right)\left(r_{c}(1-D)^{2}+4 D^{2}\right)^{2}}+\ldots \\
& \gamma_{2}^{(3)}=\frac{\omega L(1+3 D)\left(2 D+j \sqrt{r_{c}}(D-1)\right)}{r_{c}(1-D)^{2}+4 D^{2}} \beta^{-1}-\frac{r \omega L\left(F 1 r+j \sqrt{r_{c}} F 1 i\right)}{9\left(3 D^{2}-1\right)\left(r_{c}(1-D)^{2}+4 D^{2}\right)^{2}}+\ldots \\
& \gamma_{3}^{(3)}=\frac{\omega L(1+3 D)\left(r_{c}(1-D)+2 D j \sqrt{r_{c}}\right)}{r_{c}(1-D)^{2}+4 D^{2}} \beta^{-1}+\frac{r \omega L\left(F 2 r-2 j \sqrt{r_{c}} F 2 i\right)}{9\left(3 D^{2}-1\right)\left(r_{c}(1-D)^{2}+4 D^{2}\right)^{2}}+\ldots
\end{aligned}
$$

where

$$
\begin{aligned}
& F 1 r=-2\left((1-D)^{3}\left(9 D^{2}-5\right) r_{c}^{2}-f r_{1}(D) r_{c}+4 D^{2} f r_{2}(D)\right) \\
& F 1 i=f r_{3}(D) r_{c}+f r_{4}(D) \\
& f r_{1}(x)=(1-x)\left(81 x^{5}-18 x^{4}-36 x^{3}-14 x^{2}-x+4\right) \\
& f r_{2}(x)=4 x^{2}\left(81 x^{4}-9 x^{3}-45 x^{2}+5 x+4\right) \\
& f r_{3}(x)=(3 x+1)(x-1)\left(9 x^{2}-6 x-1\right) \\
& f r_{4}(x)=4 x\left(27 x^{3}-18 x^{2}-15 x+8\right) \\
& F 2 r=f r_{5}(D) r_{c}^{2}+f r_{6}(D) r_{c}+16 D^{3}\left(9 D^{2}-5\right) \\
& F 2 i=f r_{7}(D) r_{c}+f r_{8}(D) \\
& f r_{5}(x)=(x-1)^{2}\left(81 x^{4}-18 x^{3}-54 x^{2}-2 x+9\right) \\
& \left.F(x) x^{5}-117 x^{4}-72 x^{3}+86 x^{2}+19 x-13\right)
\end{aligned}
$$

48 


$$
\begin{aligned}
& f r_{7}(x)=(x-1)\left(81 x^{5}+27 x^{4}-36 x^{3}-48 x^{2}-x+9\right) \\
& f r_{8}(x)=4 x^{2}\left(81 x^{4}-36 x^{2}-1\right)
\end{aligned}
$$

The general solution of (5) is a linear combination of eigenvectors and can be written as

$$
X^{(1)}(k)=Q_{1} \lambda_{1}^{k}\left(\begin{array}{c}
1 \\
\gamma_{2}^{(1)} \\
\gamma_{3}^{(1)}
\end{array}\right)+Q_{2} \lambda_{2}^{k}\left(\begin{array}{c}
1 \\
\gamma_{2}^{(2)} \\
\gamma_{3}^{(2)}
\end{array}\right)+Q_{3} \lambda_{3}^{k}\left(\begin{array}{c}
1 \\
\gamma_{2}^{(3)} \\
\gamma_{3}^{(3)}
\end{array}\right)
$$

where $Q_{1}, Q_{2}$ and $Q_{3}$ are arbitrary constants.

Now a particular solution of the homogeneous system (a solution for zero supply voltage) may be found. Suppose that for the initial time instant $k=0$, the inductor current equals $i_{0}$ and the capacitors voltages $v 1_{0}$ and $v 2_{0}$. Then (36) can be represented as

$$
\left(\begin{array}{c}
i_{0} \\
v 1_{0} \\
v 2_{0}
\end{array}\right)=Q_{1}\left(\begin{array}{c}
1 \\
\gamma_{2}^{(1)} \\
\gamma_{3}^{(1)}
\end{array}\right)+Q_{2}\left(\begin{array}{c}
1 \\
\gamma_{2}^{(2)} \\
\gamma_{3}^{(2)}
\end{array}\right)+Q_{3}\left(\begin{array}{c}
1 \\
\gamma_{2}^{(3)} \\
\gamma_{3}^{(3)}
\end{array}\right)
$$

Resolving equation (37) with respect to $Q_{1}, Q_{2}$ and $Q_{3}$ yields

$$
\begin{aligned}
& Q_{1}=i_{0}-\frac{2 D v 1_{0}+(1-D) v 2_{0}}{(1+3 D) \omega L} \beta+O\left(\beta^{2}\right) \\
& Q_{2}=\left(\frac{D}{(1+3 D) \omega L}-j \frac{(1-D) \sqrt{r_{c}}}{2(1+3 D) \omega L}\right) \nu 1_{0} \beta+\left(\frac{1-D}{2 \omega L(1+3 D)}+j \frac{D}{(1+3 D) \omega L \sqrt{r_{c}}}\right) \nu 2_{0} \beta+O\left(\beta^{2}\right) \\
& Q_{3}=\left(\frac{D}{(1+3 D) \omega L}+j \frac{(1-D) \sqrt{r_{c}}}{2(1+3 D) \omega L}\right) \nu 1_{0} \beta+\left(\frac{1-D}{2 \omega L(1+3 D)}-j \frac{D}{(1+3 D) \omega L \sqrt{r_{c}}}\right) \nu 2_{0} \beta+O\left(\beta^{2}\right)
\end{aligned}
$$

Let us represent the eigenfunctions in a trigonometric form using equation (26) for complex conjugate roots:

$$
\lambda_{2,3}^{k}=M^{k}(\cos (k \varphi) \mp j \cdot \sin (k \varphi))
$$

Substituting equations (38), (39) and (40) into equation (36) and accounting for equation (41) yields

$$
\begin{aligned}
& i_{L}(k)=F_{11} \lambda_{1}^{k}+F_{12} M^{k} \cos (k \varphi)+F_{13} M^{k} \sin (k \varphi) \\
& v_{C_{1}}(k)=F_{21} \lambda_{1}^{k}+F_{22} M^{k} \cos (k \varphi)+F_{23} M^{k} \sin (k \varphi) \\
& v_{C_{2}}(k)=F_{31} \lambda_{1}^{k}+F_{32} M^{k} \cos (k \varphi)+F_{33} M^{k} \sin (k \varphi)
\end{aligned}
$$


where

$$
\begin{aligned}
& F_{11}=i_{0}-\frac{2 D v 1_{0}+(1-D) v 2_{0}}{(1+3 D) \omega L} \beta+O\left(\beta^{2}\right) \\
& F_{12}=\frac{2 D \beta}{(1+3 D) \omega L} v 1_{0}+\frac{(1-D) \beta}{(1+3 D) \omega L} v 2_{0}+O\left(\beta^{2}\right) \\
& F_{13}=\frac{(D-1) \sqrt{r_{c}}}{(1+3 D) \omega L} \beta v 1_{0}+\frac{2 D}{(1+3 D) \omega L \sqrt{r_{c}}} \beta v 2_{0}+O\left(\beta^{2}\right) \\
& F_{21}=-\frac{2 D \omega L}{1+3 D}\left(1+r^{2}\right) \beta i_{0}+O\left(\beta^{2}\right) \\
& F_{22}=\frac{2 D \omega L}{1+3 D}\left(1+r^{2}\right) \beta i_{0}+v_{C_{1}}(0)+O\left(\beta^{2}\right) \\
& F_{23}=\frac{(1-D) \omega L \sqrt{r_{c}}}{1+3 D}\left(1+r^{2}\right) \beta i_{0}+\frac{1}{\sqrt{r_{c}}} v 2_{0}-\frac{2 r\left(9 D^{2}-5\right) \beta}{9(1+3 D)\left(3 D^{2}-1\right) \sqrt{r_{c}}}\left(\left(r_{c}-1\right) v 1_{0}+v 2_{0}\right)+O\left(\beta^{2}\right), \\
& F_{31}=\frac{(D-1) \omega L r_{c}}{1+3 D}\left(1+r^{2}\right) \beta i_{0}+O\left(\beta^{2}\right) \\
& F_{32}=\frac{(1-D) \omega L r_{c}}{1+3 D}\left(1+r^{2}\right) \beta i_{0}+v 2_{0}+O\left(\beta^{2}\right) \\
& F_{33}=-\frac{2 D \omega L \sqrt{r_{c}}\left(1+r^{2}\right) \beta}{1+3 D} i_{0}-\sqrt{r_{c}} v 1_{0}+\frac{2 r(3 D+1)\left(9 D^{2}-5\right) \beta}{9(1+3 D)\left(3 D^{2}-1\right) \sqrt{r_{c}}}\left(\left(1-r_{c}\right) v 2_{0}+r_{c} v 1_{0}\right)+O\left(\beta^{2}\right)
\end{aligned}
$$

A particular solution of the non-homogeneous system of equations or, in other words, a partial transient process may be obtained if the steady-state values of the inductor current and capacitor voltages are known. These values exist because, as noted above, the system is stable.

To obtain the steady-state values, first, find vector $B^{(1)}$ according to equation (4). By accomplishing the operations in equation (4) and making a series expansion, vector $B^{(1)}$ is found as

$$
B^{(1)}=\left(\begin{array}{c}
\frac{(-1+3 D)}{\omega L(1+3 D)} \beta-\frac{r(-1+3 D)^{2}}{3 \omega L(1+3 D)^{2}} \beta^{2}+O\left(\beta^{3}\right) \\
-\frac{(-1+3 D)^{2}\left(1+r^{2}\right)}{2(1+3 D)^{2}} \beta^{2}-\frac{r\left(1+r^{2}\right)(-1+3 D)^{3}}{3(1+3 D)^{3}} \beta^{3}+O\left(\beta^{4}\right) \\
\frac{r_{c}\left(1+r^{2}\right)(-1+3 D)^{2}}{2(1+3 D)^{2}} \beta^{2}+\frac{r_{c} r\left(1+r^{2}\right)(-1+3 D)^{3}}{3(1+3 D)^{3}} \beta^{3}+O\left(\beta^{4}\right)
\end{array}\right)
$$


The steady-state values of the vector $X^{(1)}$ coordinates can be obtained from equation (36) for $k$ striving to $\infty$. Let us denote the vector to be found as $X^{(1)}(\infty)$. As the partial transient tends to zero, for sufficiently large $k$, the vector does not vary and equals to $X^{(2)}(\infty)$.

$$
X^{(1)}(\infty)=A^{(1)} X^{(1)}(\infty)+B^{(1)} V / 2
$$

From equation (46), $X^{(1)}(\infty)$ is represented as

$$
X^{(1)}(\infty)=\left(\begin{array}{c}
i_{L}(\infty) \\
v_{C_{1}}(\infty) \\
v_{C_{2}}(\infty)
\end{array}\right)=\left(E-A^{(1)}\right)^{-1} B^{(1)} V / 2
$$

Calculation of vector $X^{(1)}(\infty)$ from equation (47) followed by the power series expansion yields

$$
X^{(1)}(\infty)=\left(\begin{array}{c}
\frac{D}{R}+r \frac{3 D-1}{3 R} \beta+O\left(\beta^{2}\right) \\
\frac{2}{3}-\frac{\left(1+r^{2}\right) D^{2}}{(1+3 d) r} \beta+O\left(\beta^{2}\right) \\
\frac{4}{3}+\frac{r_{c}\left(1+r^{2}\right) D(D-1)}{2(1+3 d) r} \beta+O\left(\beta^{2}\right)
\end{array}\right) V / 2
$$

The non-homogeneous system particular solution is obtained by the replacement of initial conditions $-i_{0}$ by $i_{0}-i_{L}(\infty), v 1_{0}$ by $v 1_{0}-v_{C_{1}}(\infty)$ and $v 2_{0}$ by $v 2_{0}-v_{C_{2}}(\infty)-$ in $Q_{1}, Q_{2}$ and $Q_{3}$ (formula (36)) and the addition of the term $X^{(1)}(\infty)$ on the right side.

Just as for large $D$, it is interesting to consider the FCC power-up for zero initial conditions. Corresponding calculations lead to the following expressions:

$$
\begin{aligned}
& i_{L}(k)=i_{L}(\infty)+I_{1} \lambda_{1}^{k}+I_{2} M^{k} \cos (k \varphi)+I_{3} M^{k} \sin (k \varphi) \\
& v_{C_{1}}(k)=v_{C_{1}}(\infty)+U_{1} \lambda_{1}^{k}+U_{2} M^{k} \cos (k \varphi)+U_{3} M^{k} \sin (k \varphi) \\
& v_{C_{2}}(k)=v_{C_{2}}(\infty)+U_{3} \lambda_{1}^{k}+U_{4} M^{k} \cos (k \varphi)+U_{5} M^{k} \sin (k \varphi)
\end{aligned}
$$

where up to small values of the first order

$$
\begin{aligned}
& I_{1}=\left(-\frac{D}{R}+\frac{3 r\left(1-D^{2}\right)}{R(1+3 D)} \beta\right) \frac{V}{2} \\
& I_{2}=-\frac{8 r}{3 R(1+3 D)} \beta \frac{V}{2}
\end{aligned}
$$




$$
\begin{aligned}
& I_{3}=\frac{4 r\left(4 D-r_{c}(1-D)\right)}{3 R \sqrt{r_{c}}(1+3 D)} \beta \frac{V}{2} \\
& U_{1}=\frac{\left(1+r^{2}\right) D^{2}}{r(1+3 D)} \beta \frac{V}{2} \\
& U_{2}=-\frac{V}{3} \\
& U_{3}=\left(-\frac{4}{3 \sqrt{r_{c}}}-\frac{4\left(9 D^{2}-5\right)\left(r_{c}+1\right) r}{27 \sqrt{r_{c}}\left(1-3 D^{2}\right)(1+3 D)} \beta\right) \frac{V}{2} \\
& U_{4}=r_{c} \frac{\left(1+r^{2}\right) D(1-D)}{2 r(1+3 D)} \beta \frac{V}{2} \\
& U_{5}=-\frac{2 V}{3} \\
& U_{6}=\left(\frac{2 \sqrt{r_{c}}}{3}-\frac{4\left(9 D^{2}-5\right) r\left(r_{c}-2\right)}{27 \sqrt{r_{c}}(1+3 D)\left(1-3 D^{2}\right)} \beta\right) \frac{V}{2}
\end{aligned}
$$

and $i_{L}(\infty), v_{C_{1}}(\infty)$ and $v_{C_{2}}(\infty)$ are taken from equation (48).

The obtained partial transient corresponds to the time interval 1 as the first one of the PWM period. Other five possible partial transients are calculated in a similar way using the cyclic permutations of the factor indices in formulas (3) and (4). The results of other partial transient calculations are represented in the following. Let us first define the auxiliary functions:

$$
\begin{aligned}
& s_{1}(m)=\left\{\begin{array}{l}
1, m=1,2,6 \\
-1, m=3,4,5
\end{array}\right. \\
& s_{2}(m)=\left\{\begin{array}{l}
m+4, \quad m=1,2 \\
m-2, \quad m=3,4,5,6
\end{array}\right. \\
& \operatorname{off}_{1}(m)=\left\{\begin{array}{l}
\frac{3\left(1-D^{2}\right)}{1+3 D}, \quad m=1,4 \\
\frac{(1-3 D)(1-D)}{1+3 D}, \quad m=2,3 \\
1+D, \quad m=5,6
\end{array}\right. \\
& \operatorname{off}_{2}(m)=\left\{\begin{array}{c}
\frac{2}{1+3 D}, \quad m=1,4 \\
\frac{1-3 D}{1+3 D}, \quad m=2,3 \\
1, \quad m=5,6
\end{array}\right.
\end{aligned}
$$




$$
\begin{aligned}
& \operatorname{off}_{3}(m)= \begin{cases}-4 D+(-1)^{m+1}\left(1+(-1)^{m} D\right), & m=1,4 \\
2(-1)^{m+1}\left(1+(-1)^{m+1} D\right) r_{c}, & m=2,5 \\
2(-1)^{m+1}\left(1+(-1)^{m+1} D\right)+2 r D, \quad m=3,6\end{cases} \\
& \operatorname{off}_{4}(m)= \begin{cases}\frac{-D(1+D)}{2}, & m=2,3 \\
\frac{D(1-D)}{2}, & m=5,6\end{cases}
\end{aligned}
$$

Then

$$
\begin{aligned}
& i_{L}^{(n)}(k)=i_{L}^{(n)}(\infty)+I_{1}^{(n)} \lambda_{1}^{k}+M^{k}\left(I_{2}^{(n)} \cos (k \varphi)+I_{3}^{(n)} \sin (k \varphi)\right) \\
& v_{C_{1}}^{(n)}(k)=v_{C_{1}}^{(n)}(\infty)+U_{1}^{(n)} \lambda_{1}^{k}+M^{k}\left(U_{2}^{(n)} \cos (k \varphi)+U_{3}^{(n)} \sin (k \varphi)\right) \\
& v_{C_{2}}^{(n)}(k)=v_{C_{2}}^{(n)}(\infty)+U_{4}^{(n)} \lambda_{1}^{k}+M^{k}\left(U_{5}^{(n)} \cos (k \varphi)+U_{6}^{(n)} \sin (k \varphi)\right)
\end{aligned}
$$

where up to small values of the first order

$$
\begin{aligned}
& I_{1}^{(n)}=\left(-\frac{D}{R}+r \frac{s_{1}(n) \operatorname{off}_{1}(n)}{R} \beta\right) \frac{V}{2} \\
& I_{2}^{(n)}=-4 r \frac{\operatorname{off}_{2}(n) s_{1}(n)}{3 R} \beta \frac{V}{2} \\
& I_{3}^{(n)}=4 r \frac{\operatorname{off}_{3}(n)}{3 R \sqrt{r_{c}}(1+3 D)} \beta \frac{V}{2} \\
& U_{1}^{(n)}=\frac{\left(1+r^{2}\right) \operatorname{off}_{4}(m)}{r(1+3 D)} \beta \frac{V}{2} \\
& U_{2}^{(n)}=-\frac{V}{3} \\
& U_{3}^{(n)}=\left(-\frac{4}{3 \sqrt{r_{c}}}-\frac{4\left(9 D^{2}-5\right)\left(r_{c}+1\right) r}{27 \sqrt{r_{c}}\left(1-3 D^{2}\right)(1+3 D)} \beta\right) \frac{V}{2} \\
& U_{4}^{(n)}=\frac{r_{c}\left(1+r^{2}\right) \operatorname{off}_{4}\left(s_{2}(n)\right)}{r(1+3 D)} \beta \frac{V}{2}
\end{aligned}
$$




$$
\begin{aligned}
& U_{5}^{(n)}=-\frac{2 V}{3} \\
& U_{6}^{(n)}=\left(\frac{2 \sqrt{r_{c}}}{3}-\frac{4\left(9 D^{2}-5\right)\left(r_{c}-2\right) r}{27 \sqrt{r_{c}}\left(1-3 D^{2}\right)(1+3 D)} \beta\right) \frac{V}{2} \\
& i_{L}^{(n)}(\infty)=\left(\frac{D}{R}+\frac{(-1)^{n-1} r(1-3 D)}{3 R} \beta\right) \frac{V}{2} \\
& v_{C_{1}}^{(n)}(\infty)=\left(\frac{2}{3}-\frac{\left(1+r^{2}\right) \text { offs }_{4}(n)}{r(1+3 D)} \beta\right) \frac{V}{2} \\
& v_{C_{2}}^{(n)}(\infty)=\left(\frac{4}{3}-\frac{r_{c}\left(1+r^{2}\right) \text { off }_{4}\left(s_{2}(n)\right)}{r(1+3 D)} \beta\right) \frac{V}{2}
\end{aligned}
$$

The process of partial transients continuation from the discrete points of time to the entire time axis is the same as for large $D$. It involves the replacement of functions $\lambda^{k}$ by exponential terms with decay factors $\sigma=\ln (\lambda) / T_{\mathrm{PWM}}$ and trigonometric functions argument $k \varphi$ by $\Omega t$, where $\Omega=\varphi / T_{\mathrm{PWM}}$. Substitution of $\lambda_{1}$ from equation (24) and $M$ from equation (27) followed by the power series expansion yields the expressions (55) and (56):

$$
\begin{aligned}
& \sigma_{1}=\frac{1}{T_{\mathrm{PWM}}}\left(-\frac{12 r}{1+3 D} \beta-\frac{4 r\left(1+r^{2}\right)\left(9 D^{2}-5\right)\left(r_{c}+1\right)}{3(1+3 D)^{3}} \beta^{3}+O\left(\beta^{4}\right)\right) \\
& \sigma_{2}=\frac{1}{T_{\mathrm{PWM}}}\left(\frac{2 r\left(1+r^{2}\right)\left(9 D^{2}-5\right)\left(r_{c}+1\right)}{3(1+3 D)^{2}} \beta^{3}+O\left(\beta^{4}\right)\right) .
\end{aligned}
$$

\section{Natural Balancing Dynamics}

Now let us find the arithmetic mean of all six partial transients. In accordance with the definition of the FCC transient given in Reznikov et al. (2019 submitted to), this is the FCC transient. Carrying out these simple calculations and neglecting the small values of orders higher than first leads to the following expressions:

$$
\begin{aligned}
& \bar{i}_{L}(t)=\bar{i}_{L}(\infty)+\bar{I}_{1} \exp \left(\sigma_{1} t\right)+\exp \left(\sigma_{2} t\right)\left(\bar{I}_{2} \cos \Omega t+\bar{I}_{3} \sin \Omega t\right) \\
& \bar{v}_{C_{1}}(t)=\bar{v}_{C_{1}}(\infty)+\bar{U}_{1} \exp \left(\sigma_{1} t\right)+\exp \left(\sigma_{2} t\right)\left(\bar{U}_{2} \cos \Omega t+\bar{U}_{3} \sin \Omega t\right) \\
& \bar{v}_{C_{2}}(t)=\bar{v}_{C_{2}}(\infty)+\bar{U}_{4} \exp \left(\sigma_{1} t\right)+\exp \left(\sigma_{2} t\right)\left(\bar{U}_{5} \cos \Omega t+\bar{U}_{6} \sin \Omega t\right)
\end{aligned}
$$


where

$$
\begin{aligned}
& \bar{i}_{L}(\infty)=\frac{D}{2 R} V \\
& \bar{I}_{1}=-\frac{D}{2 R} V \\
& \bar{I}_{2}=0 \\
& \bar{I}_{3}=0 \\
& \bar{v}_{C_{1}}(\infty)=\frac{V}{3} \\
& \bar{U}_{1}=0 \\
& \bar{U}_{2}=-\frac{V}{3} \\
& \bar{U}_{3}=\left(-\frac{2}{3 \sqrt{r_{c}}}-\frac{2 r\left(r_{c}+1\right)\left(9 D^{2}-5\right) \beta}{27 \sqrt{r_{c}}(1+3 D)\left(3 D^{2}-1\right)}\right) V \\
& \bar{v}_{C_{2}}(\infty)=\frac{2 V}{3} \\
& \bar{U}_{4}=0 \\
& \bar{U}_{5}=-\frac{2 V}{3} \\
& \bar{U}_{6}=\left(\frac{\sqrt{r_{c}}}{3}+\frac{2 r\left(r_{c}-2\right)\left(9 D^{2}-5\right) \beta}{27 \sqrt{r_{c}}(1+3 D)\left(3 D^{2}-1\right)}\right) V
\end{aligned}
$$

After substituting $\beta, r_{c}$ and $r$ values

$$
\begin{aligned}
& \bar{i}_{L}(t)=\frac{D}{2 R} V\left(1-\exp \left(\sigma_{1} t\right)\right) \\
& \bar{v}_{C_{1}}(t)=\frac{V}{3}\left(1-\exp \left(\sigma_{2} t\right)\left(\cos \Omega t+\sqrt{\frac{C_{2}}{C_{1}}}\left(2+\frac{\left(C_{1}+C_{2}\right)\left(9 D^{2}-5\right) R T_{\mathrm{PWM}}}{54 L C_{2}\left(3 D^{2}-1\right)}\right) \sin \Omega t\right)\right) \\
& \bar{v}_{C_{2}}(t)=\frac{2 V}{3}\left(1-\exp \left(\sigma_{2} t\right)\left(\cos \Omega t-\sqrt{\frac{C_{1}}{C_{2}}}\left(\frac{1}{2}+\frac{\left(C_{1}-2 C_{2}\right)\left(9 D^{2}-5\right) R T_{\mathrm{PWM}}}{108 L C_{1}\left(3 D^{2}-1\right)}\right) \sin \Omega t\right)\right)
\end{aligned}
$$

where

$$
\sigma_{1}=-\frac{R}{L}
$$




$$
\begin{aligned}
& \sigma_{2}=\frac{R T_{\mathrm{PWM}}^{2}\left(C_{1}+C_{2}\right)}{648 L^{2} C_{1} C_{2}}\left(9 D^{2}-5\right) \\
& \Omega=\frac{T_{\mathrm{PWM}}\left(1-3 D^{2}\right)}{12 L \sqrt{C_{1} C_{2}}}
\end{aligned}
$$

The values of $\sigma_{1}$ and $\sigma_{2}$ correspond to the time constants found in Reznikov and Ruderman (2009) (see formulas (20) and (24)).

\section{Discussion}

In Figure 2, the simulation results are compared with theoretical ones for set 1 of parameters: $L=0.0004 \mathrm{H}$, $C_{1}=0.0002 \mathrm{~F}, C_{2}=0.0001 \mathrm{~F}, R=1 \Omega, T_{P W M}=0.0001 \mathrm{~s}, D=0.5$ and $V=100 \mathrm{~V}$. In Figure 3, similar comparison is being made for set 2 of parameters: $L=0.0001 \mathrm{H}, C_{1}=0.0006 \mathrm{~F}, C_{2}=0.0004 \mathrm{~F}, R=0.6 \Omega, T_{P W M}=0.0005 \mathrm{~s}, D=0.15$ and $V=100 \mathrm{~V}$.

(a)

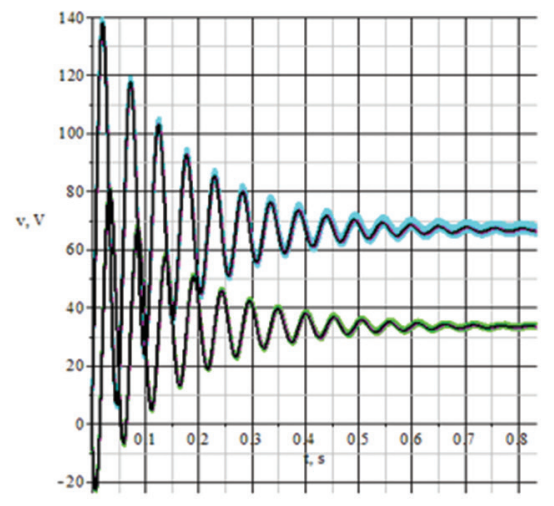

(b)

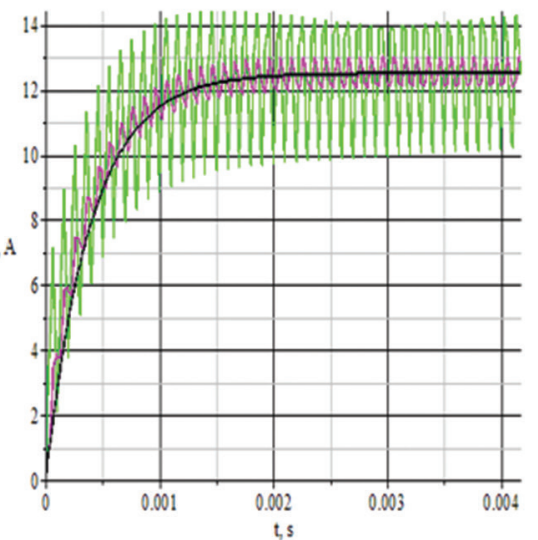

Fig. 2. The capacitor voltage transients (a) and the inductor current transients (b) for set 1.

(a)

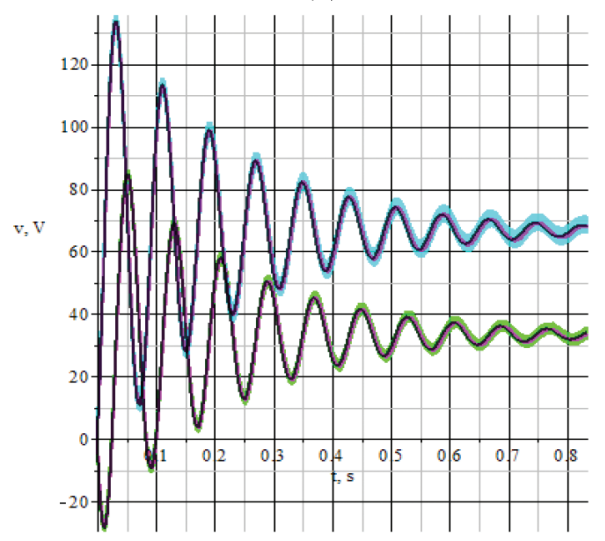

(b)

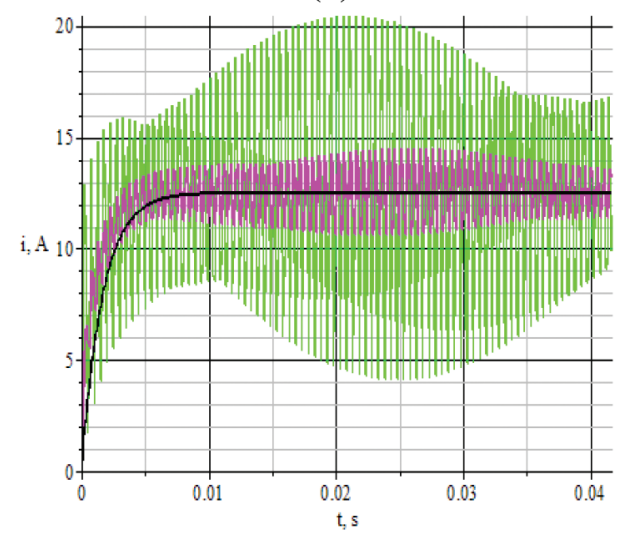

Fig. 3. The capacitor voltage transients (a) and the inductor current transients (b) for set 2. 
Figures $2 \mathrm{a}$ and $3 \mathrm{a}$ show the graphs of the capacitor voltage simulation (the green curve for $v_{C_{1}}$ and the light blue curve for $v_{C_{2}}$ ) and the transition process calculated by formulas (61) and (62) (the black curves). Figures $2 \mathrm{~b}$ and $3 \mathrm{~b}$ show similar curves for the inductor current. The black curve is calculated by formula (60). Magenta curves, which serve by references for comparison with theoretic results, on all the pictures correspond to the simulation curves filtered by a first-order low-pass filter with a time constant $T_{f}$. For the voltage curves, the time constant was selected as $T_{f}=T_{P W M}$ for the current curve $-T_{f}=T_{P W M} / 2$. All the figures show good agreement between the simulation and calculation results.

Despite the overall third order of the system, the inductor current transient practically does not differ much from the pure exponential curve and the capacitor voltage transients - from the damped oscillating curves typical for second-order system transients. The capacitor voltage transients decay much slower in comparison with the inductor current one. For instance, for the set 1 of parameters, $\sigma_{1}=-2500 \mathrm{~s}^{-1}$ and $\sigma_{2}=-6.42 \mathrm{~s}^{-1}$. For the set 2 of parameters, $\sigma_{1}=-600 \mathrm{~s}^{-1}$ and $\sigma_{2}=-4.627 \mathrm{~s}^{-1}$. The average current steady-state value amounts to $\bar{i}_{L}(\infty)=V D / 2 R=25 A^{-1}$ for the set 1 and $12.5 \mathrm{~A}$ for the set 2 . The same for the capacitor voltages: $\bar{v}_{C_{1}}(\infty)=V / 3=33.3 \mathrm{~V}$ and $\bar{v}_{C_{2}}(\infty)=2 \mathrm{~V} / 3=66.7 \mathrm{~V}$ for both sets. By inspection, the transients for large $D$ values interval $1 / 3 \leq D \leq 1$ (Reznikov et al., 2019 submitted to) and for the small values one $0 \leq D \leq 1 / 3$ coincide for $D=1 / 3$, which separates the two ranges. So, the sanity check for the continuity is an additional evidence of the correctness of theoretical calculations.

\section{Conclusion}

The article presents the time domain analysis of the four-level DC-DC FCC for small values of reference voltage $D, 0 \leq D \leq 1 / 3$. Theoretical average balancing dynamics curves obtained by formulas (60)-(62) practically coincide with the accurate simulation ones with the fast "short" time oscillations averaged out by a low-pass filter. Simple theoretical formulas (60)-(62) accurately describe the averaged "long" time processes in the 4-level FCC for small $D$ values and coincide with corresponding formulas for large $D$ values at the separating point $D=1 / 3$.

\section{References}

Reznikov, B. and Ruderman, A. (2009). Four-level single-leg flying capacitor converter voltage balance dynamics analysis. In: Proceedings of 13th European Conference on Power Electronics and Applications, Barcelona (Spain), September 2009, pp. 1-10.

Reznikov, B., Ruderman, A. and Galanina, V. (2019). Analysis of Transients in a Three-Level
DC-DC Flying Capacitor Converter. Time Domain Approach. Power Electronics and Drives, 4(39), pp. 33-45.

Reznikov, B., Ruderman, A. and Galanina, V. (2020). Analysis of Transients in a 4-Level Flying Capacitor Converter: Time Domain Approach. Part 1. Large Normalized Voltage Command. Power Electronics and Drives, 5(40), pp. 41-57. 
